Cinémas

Revue d'études cinématographiques

Journal of Film Studies

\title{
Les nouvelles écritures francophones des cinéastes afro-européens
}

\section{Olivier Barlet}

Volume 11, numéro 1, automne 2000

Écritures dans les cinémas d’Afrique noire

URI : https://id.erudit.org/iderudit/024837ar

DOI : https://doi.org/10.7202/024837ar

Aller au sommaire du numéro

Éditeur(s)

Cinémas

ISSN

1181-6945 (imprimé)

1705-6500 (numérique)

Découvrir la revue

Citer cet article

Barlet, O. (2000). Les nouvelles écritures francophones des cinéastes afro-européens. Cinémas, 11(1), 113-132. https://doi.org/10.7202/024837ar

\section{Résumé de l'article}

Comment filmer la vie? Le Tchadien Mahamat Saleh Haroun pose la question dans Bye bye Africa (1998). Il n'a pas la réponse et ne peut que tenter des explorations. Un film à tiroirs, c'est ainsi qu'il définit son film. Devant les déchirures modernes, les cinéastes d'Afrique ne peuvent que tirer des tiroirs bourrés de questions, d'incertitude et de doute. Ceux que tire Haroun dans Bye bye Africa me semblent emblématiques d'une quête thématique et donc esthétique très fortement présente dans les films récents des cinéastes francophones d'origine africaine vivant en Europe : la mémoire et la responsabilité. 


\section{Les nouvelles écritures francophones des cinéastes afro-européens}

\section{Olivier Barlet}

\section{RÉSUMÉ}

Comment filmer la vie? Le Tchadien Mahamat Saleh Haroun pose la question dans Bye bye Africa (1998). Il n'a pas la réponse et ne peut que tenter des explorations. Un film à tiroirs, c'est ainsi qu'il définit son film. Devant les déchirures modernes, les cinéastes d'Afrique ne peuvent que tirer des tiroirs bourrés de questions, d'incertitude et de doute. Ceux que tire Haroun dans Bye bye Africa me semblent emblématiques d'une quête thématique et donc esthétique très fortement présente dans les films récents des cinéastes francophones d'origine africaine vivant en Europe : la mémoire et la responsabilité.

\section{ABSTRACT}

How should life be filmed? Chad filmmaker Mahamat Saleh Haroun asks the question in Bye bye Africa (1998). He does not have the answer and can only attempt and explore the issue. He defines his film as a film à tiroirs: in the face of contemporary dissensions, African filmmakers can only open drawers crammed with questions, uncertainty and doubt. The ones Haroun opens in Bye bye Africa seem to me emblematic of a thematic, and therefore aesthetic quest very strongly present in recent films by francophone filmmakers of African origin now living in Europe: memory and responsibility.

\section{L'intimité}

À son père qui lui demande le sens de son métier, Haroun répond: "C'est pour la mémoire que je fais du cinéma." Et il cite 
Godard: «Le cinéma fabrique des souvenirs.» C'est à la recherche du souvenir que part Haroun en revenant au pays à la mort de sa mère: pèlerinage d'un cinéaste qui ne lâche pas sa caméra, le film se fait enquête sur l'état des salles à N'Djaména autant que réflexion sur le cinéma. Il doublera le regard: à celui du film, caméra invisible, s'ajoutera celui de sa propre caméra, image vidéo en noir et blanc. Le procédé n'est pas neuf; il permet l'irruption d'un autre temps du cinéma, celui de l'improvisé et de l'amateurisme qui tournent le dos au prévu et au professionnel. De cette opposition parfaitement construite naît l'impression de spontanéité recherchée dans ce genre très prisé aujourd'hui qu'est le documentaire-fiction. Et c'est justement en mêlant les registres que Haroun entend poser les termes de sa réflexion.

Documentaire sur une ville d'où le cinéma a été chassé par la vidéo et par la guerre, recherche de ses derniers défenseurs, balade posthume dans des salles dévastées, le film se fait également fiction lorsqu'un cinéaste retrouve un ancien amour de tournage. C'est en abordant ainsi l'intime, le privé, qu'il trouve le mieux son souffle. Mettant en scène le désir, il suit le parcours des caresses en des moments de pure émotion visuelle, conjuguant le gros plan, des scènes d'approche en vidéo, une caméra à l'épaule incertaine, louvoyant dans les diagonales avant de cadrer le sujet.

Aussi, quand il faudra mettre le préservatif avant de s'unir, le retour à la réalité n'en sera-t-il que plus dur. De plus, Isabelle demande au cinéaste de l'emmener : elle a tenu le rôle d'une malade du sida dans son film précédent et, le public faisant mal la différence, son entourage l'a rejetée. La réalité de l'acteur en Afrique sert ainsi de toile de fond à une confrontation dont le cinéaste ne sort pas grandi : il l'envoie promener, disant qu'elle lui prend trop la tête. C'est cette capacité à se mettre en danger, à prendre des risques dans la forme comme dans le fond, à poser des questions sans réponses, à explorer l'humain sans concession, qui fonde une nouvelle écriture d'autant plus réjouissante qu'elle actualise une des fonctions essentielles du cinéma: aider chacun à se regarder en face pour la responsabilisation de tous.

En bonne logique, ce cinéma travaille avant tout l'improvisation, tant dans l'image que dans le jeu des acteurs, les détails 
quotidiens qui font sourire, le témoignage direct face à la caméra, notamment pendant les scènes de casting, la personnalisation du propos et l'implication de l'énonciateur. Ce cinéma de mémoire est ainsi celui de la sincérité et sans doute le meilleur cadeau à faire au spectateur. Haroun finit d'ailleurs par offrir sa caméra à son jeune cousin pour qu'il filme la famille et comble la distance de l'exil: lui aussi pourra filmer la vie avec ce regard qui sait dire "ça me regarde»...

Le premier geste de nombreux cinéastes a été d'interroger leur origine. Tanun (1994), du Guinéen Gahité Fofana, est ainsi une rencontre intime entre le cinéaste et son grand-père, un dialogue drôle et lucide entre le monde d'hier et cette caméra qui le scrute: "Ma démarche était d'utiliser le cinéma, disait encore Fofana. Notre complicité lui a permis de bien comprendre ce qu'était un film. C'est donc aussi un vieil homme qui parle de cinéma dans un village en Afrique ${ }^{1}$." Comprenant parfaitement la force de mémoire du cinéma, cet homme qui n'a jamais vu un film saisit qu'il peut ainsi s'adresser à ses descendants. On retrouve la même quête chez le Camerounais Jean-Marie Teno. Avec Hommage (1985), lui aussi a débuté par un retour au pays, les funérailles de son père lui fournissant l'occasion de renouer avec son village et de comprendre les changements survenus.

Cette génération des fils de l'Indépendance a ainsi logiquement commencé par s’interroger sur ce qui la précède, mais elle l'a fait sans la radicalité anti-autoritaire des révoltes soixantehuitardes, en alliant lucidité et sens de la filiation: "Faire un film sur mon père était l'occasion de poser, après trente ans d'indépendance, la question de notre responsabilité dans ce qui se passe en Afrique aujourd'hui " (Barlet, 1998d, p. 9), disait David Achkar à propos de son film Allab Tantou (1991).

C'est bien l'angoisse de sa filiation qui pousse un jeune métis à s'entêter dans sa poursuite de ce Mario qui sort de prison et ne veut pas entendre parler de lui. Ne porte-t-il pas malgré lui le poids de la trahison? Celle de l'amitié fraternelle qui unissait Mouss et Mario, le Noir et le Blanc, nés le même jour à la fin des années cinquante et élevés comme des frères jumeaux? Après en avoir retravaillé le montage, le Guinéen Mama Keïta a rebaptisé son film. Choisis-toi un ami est devenu Le Onzième Commande- 
ment, qui vient s'ajouter aux dix commandements bibliques: "Choisis-toi un ami, aime-le selon les commandements de l'Éternel, si tu n'en as pas la force, tue-le». Mama Keïta précise:

Ce "tue-le» qui peut paraitre brutal, qui tombe comme un couperet, c'est: tue cette amitié. Si tu ne peux pas souscrire aux dix commandements, renonce à cette amitié. Parce que le prix que tu auras à payer en cas de manquement serait un prix trop élevé à payer. C'est pour ça que je dis : tue-le. Parce que l'amitié, c'est une exigence ${ }^{2}$.

Cette génération de cinéastes n'est pas celle des compromis. Dans sa quête de mémoire, dans sa recherche de filiation, dans son exploration de l'intime allant jusqu'à l'autobiographie, elle revendique l'implication, la prise de risques, une introspection qu'elle estime nécessaire pour que l'Afrique puisse se définir un avenir, ce qu'Achkar exprime en ces termes:

L'Afrique doit chercher une certaine Rédemption. Elle doit se confronter à son propre mal. Mon film marche bien aux États-Unis auprès des jeunes étudiants qui veulent repositionner leur identité noire dans son ensemble et non seulement dans un rêve d'Afrique charriant des images d'Épinal à la Camara Laye, celles d'une Afrique imaginée qu'on a envie de retrouver (Barlet, 1998c, p. 10).

\section{Devant les préjugés}

Car tout le problème est encore aujourd'hui d'éviter ce que Césaire appelait une "sous-culture coloniale». Dans le monde du cinéma, cette impasse consisterait d'une part à jouer le mimétisme en essayant de faire du cinéma "comme les Occidentaux" et d'autre part à répondre à la demande occidentale, à privilégier l'anecdote documentaire au détriment de la vision intérieure alors même que, comme le dit Glissant, "le décor n'est pas pays". C'est justement le reproche adressé par les jeunes cinéastes à certains de leur pairs et de leurs aînés, car ils estiment le contenu des films trop téléguidé par le financement occidental:

Je préfere qu'il y ait deux films seulement, voire pas de films du tout, dit encore Keïta, mais qu'il y ait la vo- 
lonté de faire des films libres, plutôt que d'avoir une profusion d'images qui soient dictées par l'extérieur, dont les thèmes sont pensés et posés de l'extérieur, dans les commissions siégeant dans les ministères!

Le risque serait pourtant de tomber aujourd'hui dans la critique sans nuances d'une mondialisation vue comme une occidentalisation du monde visant à la mort des cultures au profit d'une pensée unique, par assimilation idéologiquement forcée ou même par destruction planifiée. Ces cinéastes, sans cacher leur colère contre les scandales du néocolonialisme, refusent la facilité d'une dénonciation bien-pensante, celle qu'Achille Mbembe dénonçait comme obstacle à l'avancée de la recherche en parlant d' "une approche émotionnelle et polémique du monde qui, souvent, se cache derrière le masque d'un radicalisme de vernis dont il faut bien reconnaitre la nature conservatrice» (Bah, 1998, p. 50).

Comment éviter ce travers? En mettant en exergue les permanentes négociations à l'œuvre entre les différentes cultures, négociations que les cinéastes vivent dans leur quotidien métissé d'exilés (souvent métis eux-mêmes). Ils en témoignent par leur refus de se laisser enfermer dans les concepts figés d'identité ou d'authenticité, récusant ainsi les discours communautaristes, culturalistes ou essentialistes idéalisant les valeurs culturelles.

Devant l'universalité dévorante de la culture dominante, la tentation de l'antiracisme fut de fonder son combat sur la défense des différences culturelles érigées en valeurs absolues. Le risque était d'enfermer l'Autre dans une place, un rôle, des valeurs mythiques. Pour s'en dégager, les immigrés en France sont passés de la revendication du "droit à la différence» dans les années quatre-vingt (avec SOS-Racisme) à celle du "droit à l'indifférence" dans la décade suivante, opposant ainsi aux discriminations les droits de monsieur Tout-le-monde, demandant également qu'on les laisse tranquilles, alors même que le discours raciste se banalise - que plus de soixante pour cent des Français se déclarent racistes dans un récent sondage inquiète non parce qu'ils sont racistes, ce qu'on savait déjà, mais parce qu'ils osent le dire ouvertement ${ }^{3}$.

Le malentendu essentiel de l' "assimilationnisme" républicain français consiste à "civiliser" tout en se préservant, donc en 
maintenant dans une position subalterne cet Autre qui se trouvera enfermé dans des stéréotypes réducteurs : naif, primitif, contemplatif, ingénu, candide, inexpérimenté, et donc finalement intellectuellement limité ${ }^{4}$. Cette contradiction fonde avec l'Autre en général et l'Africain ancien colonisé en particulier une relation faite de projections où la différence sert de repoussoir pour se forger sa propre identité en même temps qu'elle offre un moyen d'évasion, une possibilité d'échapper à la grisaille du quotidien.

La critique cinématographique ne fait pas exception, elle qui use largement de ces préjugés ${ }^{5}$. En exotisant, en folklorisant l'Autre ou, bien plus récemment, en taxant ses créations de passéisme ou d'académisme, elle l'enferme dans sa différence et s'assure qu'il y restera. Joseph Kumbela joue avec ces clichés dans un hilarant court métrage de huit minutes, Taxcarte (1997): un cinéaste africain en boubou arpente les allées d'un festival de cinéma africain, mais se retrouve pris à son propre piège lorsque ses deux conquêtes européennes non seulement s'aperçoivent qu'il leur a tu l'existence de l'autre, mais aussi apprennent que sa femme en Afrique vient d'avoir un nouveau bébé...

C'est afin d'échapper aux catégories d'authenticité, d'identité et de racine unique que les cinéastes d'origine africaine revendiquent souvent un statut indifférencié de "cinéaste tout court". Seulement, l'étiquette africaine constitue leur principal atout, non seulement pour la diffusion dans les festivals spécialisés (diffusion généralement rémunérée, mais parfois commandée par l'impossibilité de sortir leurs films en salle), mais aussi pour le financement de leurs films, car elle leur ouvre des guichets dont ne peuvent profiter les cinéastes français (Fonds Sud, Francophonie, Coopération, Union européenne, fondations, etc.).

Voilà donc les cinéastes en train de jouer un tango, louvoyant entre la revendication de leur africanité et leur volonté d'y échapper pour ne pas donner prise aux projections.

\section{De l'intime au collectif}

Cette volonté d'échapper à l'enfermement par la différence est à la source de nouveaux malentendus: risque de l'indifférenciation alors même que tout réalisateur explore ses racines, son origine, pour en dégager les spécificités qui définiront son origi- 
nalité; fantasme d'un statut indifférencié, comme s'il existait un cinéaste en soi, pouvant se tenir à l'écart des contingences culturelles et hors des rapports de force de la situation postcoloniale.

Cette volonté est une réaction contre l'enfermement et les préjugés. Pour s'en dégager, les cinéastes tendent à affirmer que leur spécificité serait justement de ne pas en avoir, la définissant plutôt comme un devenir, une quête, un entre-deux culturel en permanente mutation, une hybridité mouvante entre deux cultures.

Dès lors, ils échappent au dilemme de la différence en jouant l'intime, mais sans jamais le faire avec cette étrangeté au monde que l'on rencontre souvent dans les expressions occidentales. Au contraire, pour reprendre une remarque de Glissant (1981, p. 759), "cet intime est inséparable du devenir de la communauté ". En cela, ils poursuivent la voie tracée par leurs aînés. Cette volonté d'inscrire leur réflexion intimiste dans le réel de la mémoire de leur peuple leur permet, comme l'écrivait Gilles Deleuze (1985, p. 289), de franchir «la frontière qui séparerait son affaire privée de la politique" et de "produire des énoncés collectifs".

L'Afrique ne se trouve pas idéalisée pour mieux échapper aux exclusions vécues dans la société d'accueil. D'où cette conscience aiguë dess réalités sociales africaines, motivée par un sentiment d'appartenance: "Même si j'ai choisi de vivre en France, je ne peux oublier cette partie de moi-même qui est de là-bas, ces racines fondatrices, cette mémoire si vivace, malgré l'exil", écrit Mahamat Saleh Haroun en mars 2000 dans le premier numéro du bulletin de la Guilde africaine des réalisateurs et producteurs ${ }^{6}$. Cela commence par un constat qui imprimera au film un ton documentaire. Dans un six minutes sans concession, Elle revient quand Maman? (1998), dédié à Zao (qui chantait une Afrique "cadavrée "), David-Pierre Fila montre crûment la mort, le $1^{\text {er }}$ janvier 2000, d'une femme paludéenne à l'hôpital. Cette mère Afrique qui meurt, chacun la porte en soi. "Comment croire au cinéma dans un pays où la guerre est devenue une culture?" demande Haroun dans Bye bye Africa.

Il ne s'agit pas de simplement refléter la réalité: une certaine image du monde se construit sur l'écran, un projet politique se 
fait jour, qui tend vers un nouvel imaginaire. Ali, le chauffeur de taxi, parcourt les rues de N'Djaména, chargeant au passage toute une galerie de personnages: c'est Un taxi pour Aouzou (1994) du Tchadien Issa Serge Coelo. Le taxi est une tour d'observation privilégiée pour « remarquer les réalités politiques, la misère sociale, l'amour... qui sont des sujets ayant leur place au cinéma" (Barlet, 1995, p. 17).

Le problème de la critique est dès lors de saisir "l'esprit » d'un auteur et la culture qui l'a nourri, de saisir une vision singulière sans la détacher de son contexte énonciatif. C'est ainsi que l'on ne peut séparer la chronique d'un quartier populaire de Kinshasa que fait José Zéka Laplaine dans Macadam Tribu (1996) de l'expérience personnelle du réalisateur pour saisir comment il en extrait une famille - la mère qui boit, Mike le fils dragueur qui sort de prison, Kapa le fils boxeur dont le combat tourne mal..., mais aussi des Blancs, un Français, un Italien, et un métis - et nous entraîne avec eux dans une Afrique urbaine et profondément métissée:

Je suis né en brousse au moment de l'indépendance. Ma mère est partie s'installer avec moi à Kinshasa, qui paraissait le seul endroit sûr. J'y ai passé toute mon enfance et n'ai découvert l'Europe que lors d'un voyage à dix-sept ans. Macadam est construit sur une mosaïque de personnages mettant en valeur leurs qualités humaines. Il met en scène leur vie commune, leurs liens affectifs, cette microsociété d'un quartier africain. Le montage s'est donné pour but de rendre cette cohésion et cette diversité. [...] J'ai voulu dans le film conserver le regard de la génération des trente ans, celui des enfants Bavusi, lorsqu'ils regardent leur mère culpabilisée par la dérive de ses enfants qui n'ont pas "réussi». Elle souffre de s'être peut-être trompée dans ce mariage avec un Blanc. Elle ne doit pas être jugée et ses secrètes solitudes doivent être respectées ${ }^{7}$.

Ne les enfermons surtout pas dans les certitudes de nos jugements. Respectons-les, car leur silence est éloquent. Comme le sont les non-dits de Saïd et Rajeh, les deux amis intimes, si intimes, de Sabriya du Mauritanien Abderrahmane Sissako. Dans ce café perdu dans le sable tunisien, où le temps semble coller aux 
parties d'échecs, le hasard introduit brutalement une jeune femme... Cette intrusion de l'ailleurs restaurera le désordre. Quel est cet autre qui pousse la femme à essayer d'entraîner l'homme à partir? Quelle est cette vie qui cherche à rompre leur lien de mort? Les seuls êtres qui rôdent autour du huis-clos d'Ainsi soit-il (1997) du Sénégalais Joe Gaye Ramaka sont des enfants malades. Image-miroir de deux adultes? Rien ne semble pouvoir se résoudre, rien ne peut se construire, personne ne peut se sauver...

Comment sortir des blocages qui enferment? Les relations amoureuses (qu'explorent ces deux films de la série «Africa Dreamings ") en sont les miroirs les plus charnels, les plus criants. Les cinémas d'Afrique, sans doute par pudeur comme par devoir idéologique, les avaient peu abordées; la jeune génération s'engouffre dans la représentation du désir et rapproche sa caméra des corps, car c'est dans le désir que se situe ce corps profond du cinéma: l'affirmation du sujet, l'individuation, cette force souterraine et subversive qui pourrait permettre d'explorer sans concession la modernité ${ }^{8}$. La valse hésitante de Malou qui vient passer un week-end chez Bwési et les efforts maladroits de ce dernier pour la posséder dans La Fumée dans les yeux (1998), du Camerounais François Woukoache, témoignent de la liberté de ton de cette entreprise de responsabilisation. Le cinéaste se regarde en face, jusqu'à jouer son propre rôle dans Bye bye Africa. Il est dès lors cinéaste et tout simplement homme avant d'être Africain, sans aucunement renier son origine et sa culture. Il partage en cela la nécessité de se réapproprier son corps avec tout humain aux prises avec l'appauvrissement que constituent les modèles modernes de désincarnation représentés par les top models dans les médias.

Jouer l'intime n'est subversif qu'en s'appuyant sur la force de résistance de sa culture: l'individu n'est prophétique qu'en émanant de sa culture, en convoquant sa mémoire. Mais rien n'est simple car la mémoire est dignité, et c'est justement sa dignité qui fut volée à l'Africain... Un enfant court sur une plage sur des mots de Césaire: "Dans ma mémoire sont des lacunes. Ma mémoire a sa ceinture de cadavres...» Dès lors, que montrer? C'est effectivement par la question de l'image que commence le très fort Asientos (1995) de François Woukoache. Sur l'écran

Les nouvelles écritures francophones des cinéastes afro-européens 
d'un poste de télévision, en diagonale instable, des photographes occidentaux mitraillent l'horreur du génocide au Rwanda. L'Histoire bégaie: “c'est en train de recommencer». Le cinéaste surfe pour trouver l'image juste, éviter l'écueil du misérabilisme, sempiternelle vision de l'asservissement et de la passivité. "Pourquoi la mer ne rejette-t-elle pas ces cadavres?» De la traite, il n'y a rien à voir, rien à montrer. La caméra épouse les murs nus de Gorée. Et de nouveau la peau en gros plan, la peau ridée de ce vieil homme assis sur la plage du souvenir, comme si ces plis étaient ceux de l'Histoire. Une voix de femme, une voix intérieure, pour regarder le passé en face, pour mieux voir le présent: "Se taire, écouter le silence, réapprendre à regarder pour voir l'innommable."

La vague de cinéastes des années quatre-vingt a cherché à changer l'image de l'Afrique sans s'interroger sur le moment où cette image qu'ils récusaient avait vu le jour, déclare François Woukoache. C'est le moment de la traite où l'homme noir a été défini comme un objet par l'Autre. Asientos est un film qui s'est construit à partir de ce postulat. Il s'agissair d'exprimer quelque chose de différent qui n'ait rien à voir avec ce que j’ai déjà vu, notamment dans le cinéma africain. Il fallait qu'on sente tant au niveau du sujet que de la forme que ce film est nécessaire, sinon il n'allait servir à rien (Barlet, 1998d, p. 41).

La question de la représentation est dès lors centrale dans l'esthétique des films. Lorsqu'il représente la traite négrière pour restaurer la mémoire, le Martiniquais Guy Deslauriers, dans Le Passage du milieu (1999), casse l'image dès qu'elle pourrait tomber dans le pitoyable et le misérable: il joue de saccadés, d'images tronquées, d'un montage en à-coups, d'un parallèle persistant avec l'Afrique et ses compromissions. L'Histoire, sujette à toutes les reconstructions imaginaires, n'est pas montrable. C'est toute la différence avec Amistad: le non-dit et le non-montré sont, comme le symbole, plus proches de la réalité que la remise en scène historique, présentée comme objective bien que parfaitement idéologique et subjective, de ce regard à la Spielberg qui a horreur du vide, de Schindler à Ryan. 


\section{Le rythme de l'oralité}

Pour exister autrement qu'en objet défini par l'Occident, les cinémas d'Afrique ont besoin d'une analyse permanente, d'une preuve de leur existence, voire même de leur statut d'œuvre de cinéma, tant l'Occident leur nie la capacité de créer, les excluant bien souvent d'études approfondies dans les revues de cinéma! D'où la profusion de films sur le cinéma africain, à la faveur de cette fête du cinéma qu'est le festival de Ouagadougou - le Fespaco - qui se tient tous les deux ans. Il s'agit sans cesse de préciser le sens et la portée des films autant que les difficultés de leur gestation, à coup d'interviews de réalisateurs, de commentaires et d'extraits. Seul Les Diseurs d'histoires (1998) du Noir algérien Mohamed Soudani, par son introspection sans complaisance des problématiques des cinémas du Sud, échappe heureusement à cette nécessité anthologique en quête d'unité et de reconnaissance.

N'était-ce pas le colonisateur qui voulait enfermer l'Afrique dans une unité fictive? Et qui nous fait encore aujourd'hui considérer dans maintes manifestations l'Afrique comme un pays? qui nous empêche de préciser l'origine des réalisateurs? La dissémination et la diversité des œuvres permettent qu'elles échappent à toute catégorisation et partant à toute réduction. Une spécificité les réunit cependant: leur hybridité. «La reconnaissance d'un moi pluriel, issu d'une négociation permanente entre l'origine et la culture dominante et l'examen de son expression littéraire constitueraient une nouvelle direction des études postcoloniales ", suggère Jean-Marc Moura (1999, p. 71) ${ }^{9}$.

Les cinéastes prennent effectivement un malin plaisir à brouiller les cartes de l'identité, comme Joseph Kumbela dans L'Étranger venu d'Afrique (1998) où Lulu, un étudiant africain à Pékin, est francé avec Sun, une jeune Chinoise. Les différences culturelles y sont à l'origine de savoureux quiproquos. Explorant l'interaction générale des cultures, les cinéastes définissent une nouvelle modernité qui ne résout pas les tensions mais leur donne un sens, déconstruisant ainsi leurs antinomies.

Il s'agira donc de définir les marges, de dessiner une géographie de la périphérie, un portrait de la marginalité. Ce seront ces chroniques de l'Afrique urbaine, celle des "brigands" de Ouagadougou dans Haramuya (1995) du Burkinabè Drissa 
Touré ou celle des jeunes des cités de Yaoundé et de leur parler imagé dans Quartier Mozart (1992) du Camerounais Jean-Pierre Bekolo. Esthétiquement, ces films se doivent de sortir de la linéarité : leur montage en cercle évoque les répétitions et les parenthèses de la tradition orale (Barlet, 1996, p. 192 et 216).

Les nouvelles écritures cinématographiques opèrent ainsi un véritable retour aux sources, se saisissant de leur fonds culturel pour nourrir une esthétique appropriée aux nécessités modernes de leur discours. Balufu Bakupa-Kanyinda, originaire du Kasaï, explique ainsi comment le langage griotique du kasala marque son écriture au cinéma :

En écoutant le kasala, on est ému, mais c'est surtout en comprenant comment il fonctionne. Il ne fonctionne pas sur les émotions, il fonctionne sur les images que le narrateur suscite en toi. Les images d'un passé de bravoure, du présent, du futur; contrairement aux griots maliens, les diseurs de kasala ne glorifient pas une personne, mais installent une personne dans une lignée, ou un territoire dans une lignée. Et ça suscite des images, pas des émotions, mais l'émotion est dans l'image. J'ai donc écrit des textes comme ça, c'est-à-dire en partant d'une sculpture, très proche des kasalas, qui fonctionnent en structures tressées. En poésie, ce serait des rimes tressées, à la limite. Et j'ai fonctionné ainsi pour mon film Dix Mille Ans de cinéma, en essayant au niveau de l'image de tresser ce qui va être dit avec ce qui va être filmé - tout en créant toujours, à l'intérieur de la sphère narrative, cette tresse qui paraît être une déstructuration, mais qui devient une structuration, parce qu'elle est voulue ainsi ${ }^{10}$.

\section{Cela passe aussi par des trucs:}

La voix off devait porter un malaise poétique, être fatiguée, comme venant de loin, ayant marché. Et pour y arriver, moi-même je me mets en conflit: je me bourre au whisky, comme pour Dix Mille Ans, mais je le fais dans la salle de montage avec un walkman, pour que le son n'ait pas la texture professionnelle forte d'un DAT ou d'un Nagra. Dans Sankara, j'ai appliqué toujours ça en cabine de montage, je gardais toujours la même atmosphère mais en essayant d'être proche avec la voix 
des montées du griot. La voix off ne raconte pas vraiment le film, elle raconte ce que j'ai moi à l'intérieur sur cette histoire, mais avec des pointes proches d'un griot de l'Ouest.

Le Damier (1997), du même Balufu, met directement en scène l'oralité populaire de l'interpellation dans l'affrontement du champion d'échecs de la cité avec le dictateur - et la sacralise même puisqu'il en fait l'outil de sa victoire. Plus encore, un film comme Bye bye Africa regroupe une multitude de traits caractéristiques de la tradition orale: les approximations revendiquées de la narration qui connotent l'incertitude recherchée, les précisions et digressions comme des parenthèses dans le récit qui viennent l'éclairer, les apostrophes au spectateur par des visages de face regardant la caméra, le maintien de l'illusion d'un double regard avec la caméra vidéo marquant la présence d'un public... Autant d'appels à la participation de spectateurs constamment sollicités par le narrateur. Finalement construit comme une accumulation d'anecdotes à la grammaire complexe, combinant appositions et coordinations pour multiplier les parallélismes, le film trouve une poésie proche du chant méditatif ou plutôt du blues, forme musicale caractéristique de nombreux films, dont un des plus beaux exemples est le docu-fiction Waalo fendo (Là où la terre gèle, 1998) de Mohamed Soudani sur des immigrés sénégalais à Milan. Là encore, témoignages face à la caméra, construction du récit en tant que rythme plutôt que linéarité, images sensibles de l'environnement urbain entrecoupées de retours à l'Afrique, etc. contribuent à ponctuer le film d'interrogations en images, dans un éclatement du discours exprimant tant le métissage que la multiplicité linguistique caractéristique du vécu de l'exilé.

Dans leur refus du film de légende décrié sous l'appellation méprisante de "cinéma calebasse », ce n'est pas la réécriture des valeurs essentielles du conte que rejettent les jeunes cinéastes. Ils s'en garderaient bien, l'appel à l'origine étant à la source de leur culture et de leur spécificité. Seulement, ils refusent le compromis qu'ils jugent concédé par certains cinéastes envers une demande occidentale exotisante d'une oralité délicieusement primitive et rurale puisque seulement vue comme une contemplation, un "supplément d'âme». Ils dénoncent le racisme que 
suppose la dualisation qui apparie Nord et écriture, Sud et tradition orale, racisme d'une vision de l'Afrique qui leur semble repris dans des films où ils ne discernent pas d'engagement politique pour un changement social. Par contre, ils accueillent chaleureusement un film comme Taafa fanga (Le Pouvoir du pagne, 1997) du Malien Adama Drabo, car il se saisit d'une légende dogon pour construire un discours très actuel sur les relations entre hommes et femmes, discours qui appelle à l'égalité dans la différence - message clairement perçu par les Maliens eux-mêmes puisqu'on parle à Bamako depuis la sortie du film de "l'effet Taafa fanga" lorsque des femmes se rebellent contre les attitudes patriarcales de leurs maris.

\section{Saisir la précarité}

Cette articulation entre le récit et la communauté, entre l'individuel et le collectif, reste non seulement fondamentale dans les nouvelles écritures, mais elle passe au premier plan des préoccupations. Cela ne va pas sans reposer sans cesse la question de la réalité (qui constitue, comme le disait Gaston Kaboré, "le cœur et le corps des films").

Je ne sais pas si c'est bien, indique François Woukoache, mais je pense qu'aujourd'hui le cinéma que l'on " doit" faire si on veut avoir un public, si on veut créer un mouvement autour de l'art en général, doit repartir du documentaire. Parce que c'est la vie des gens, et quelle que soit leur culture, mais surtout en Afrique où la vie est devenue extrêmement dure et violente, les gens ont envie de rêver à partir des choses qui les touchent directement dans leur vie de tous les jours. C'est une réflexion qui mamène à une radicalisation du style et donc de la manière de faire les films. Il faut se réapproprier une autre façon de toucher ces gens; je pense que ça passe par des moyens de production plus légers et des choses plus directes. Notre "grand cinéma " viendra le moment venu ${ }^{11}$ !

Cela motive une nouvelle définition du cinéma, combinant indépendance et créativité pour offrir un autre point de vue sur la réalité, ancré dans l'expérience quotidienne: "C'est quand même la base de la société de savoir comment on vit ensemble, com- 
ment on gère les relations entre nous, comment on aime aujourd'hui...", précise encore Woukoache. Est-ce une nouvelle vague du cinéma? Il est vrai que ces cinéastes rejoignent ce qui a fait la nouvelle vague française: petits budgets, improvisation, tournages rapides en extérjeurs ou sur des lieux naturels, petites équipes, rupture avec la chronologie dans le récit, fragmentation du propos marquant le manque de cohésion de la jeunesse de l'époque...

Nous n'avons pas la prétention de nous comparer historiquement à ce qui s'est fait avec la Nouvelle Vague, réplique Jean-Marie Teno, et comme notre cinéma africain est toujours en balbutiements et en train de se renouveler, on ne peut pas dire qu'on est dans les mêmes conditions que la Nouvelle Vague. Disons seulement qu'on essaie de mettre des fondations pour un travail de réflexion qui prendra certainement beaucoup d'années, mais dont on espère qu'il amènera un esprit différent de celui qui existe actuellement, c'est-à-dire l'individualisrne qui fait qu'on passe tout notre temps à refaire souvent les mêmes erreurs que les aînés ${ }^{12}$.

Cela suppose que la sensibilité penche vers l'incertitude, la précarité, la dérision. Un peu comme lorsqu'une caméra cadre le paysage par la fenêtre d'un train: c'est l'aléatoire qui fixera ce qu'on y verra, et où elle s'arrêtera en gare. Le choix du cinéaste est davantage dans le fait de placer sa caméra face à cette fenêtre que de définir le contenu de son image. Cette charge d'arbitraire, à l'opposé de l'accumulation de signes et de clichés qui caractérise l'image cinéma des stars, pourrait définir une nouvelle écriture à l'écoute de la vie. Dans Vacances au pays (2000), Jean-Marie Teno reprend le chemin du village de son enfance et s'y retrouve au moment d'un congrès, réunion traditionnelle de tous les habitants. Le congrès qui autrefois permettait une discussion collective et ouverte sur les problèmes à résoudre et les tensions au sein de la collectivité est devenu une fête commanditée par une brasserie. Le match de foot coutumier se déroule sur un terrain dont on n'a pas réussi à couper l'herbe. Teno aurait pu accéder à la demande de son opérateur qui voulait surélever la caméra pour que l'on voie les joueurs, mais il a maintenu la hauteur choisie durant le reste du film, si bien que c'est à travers 
un rideau d'herbes que l'on suit le match! Par l'image plus que par le commentaire, il met l'accent sur la déliquescence du processus social sous le poids de la modernité. C'est ce choix esthétique sans concession de ne pas élever la caméra qui est fondamental: image du réel qui rappelle la manière godardienne de faire un plan. L'image ne répond pas à la nécessité du récit mais à la nécessité d'être là, d'avoir été filmée pour ce qu'elle est, êtres, choses et situations évoquant une profonde responsabilité, celle du cinéaste se résumant dans le choix qu'il a fait de cette image-là ${ }^{13}$.

C'est ce choix qui permet à l'émotion d'appuyer un message qui n'a pas besoin d'être dit puisque l'image le manifeste, et qui permet au film de trouver son universalité sans être universaliste.

\section{Spécificité du regard}

Est-ce cette "position passionnelle" de l'intellectuel colonisé dont parlait Fanon, cette hypersensibilité, cette sensitivité exceptionnelle qui pousse les cinéastes à adopter une esthétique poétique et lyrique de l'engagement? Ils reprennent volontiers à leur compte les paroles fortes de Césaire: Woukoache dans Asientos, le Mauritanien Abderrahmane Sissako dans La Vie sur terre (1998). Mais c'est sur le ton de la méditation et non de l'incantation entre deux termes antagoniques dont on forcerait les traits pour satisfaire aux exigences de la démonstration. Ils se distancient des positions idéologiques parfois manichéennes de leurs aînés pour n’en garder qu'une géographie de la périphérie, prenant pour sujet les marginalités de leurs sociétés dans des chroniques de la vie urbaine, celles déjà évoquées d'Haramuya ou de Quartier Mozart, ou encore celle de Dôlè (L'Argent, 2000) du Gabonais Imunga Ivanga qui s'attache aux quatre cents coups et aux rêves d'une bande de jeunes des matitis de Libreville laissés à l'abandon, comme l'avait déjà fait à ses débuts le Malien Cheick Oumar Sissoko dans Nyamanton (1986). Ou bien c'est tout simplement la marginalité de la brousse par rapport au rythme moderne que Sissako visite dans La Vie sur terre, situé au moment du passage à l'an 2000 dans un village malien où "l'intention de communiquer est plus importante que la communication elle-même" (Barlet, 1998b, p. 99). 
Cet engagement passe par une certaine humilité: "Ce que j'essaye par ce film qui est parfois un cri mais surtout un chuchotement, d'autres le font tous les jours dans leur quotidien, dit encore Sissako. Je ne suis pas au-devant des combats. Ma forme est visible mais d'autres ont plus de mérite." Il s'agit avant tout de témoigner des tensions que ressent le cinéaste tant dans sa situation d'exilé que dans ce qu'il perçoit de l'Afrique. C'est une inquiétude qui le pousse à se détacher de toute gratuité esthétique avant-gardiste pour se recentrer sur l'urgence sociale d'un continent en déroute ou sur les réalités de l'exclusion dans sa société d'accueil. C'est la liaison qu'il opère ainsi avec son origine culturelle qui font la spécificité de son regard, qu'aucun autre ne peut remplacer. Et c'est pour cela que son regard est essentiel, non par authenticité africaine mais tout simplement parce qu'il est spécifique.

Un exemple: Doulaye, une saison des pluies (1998), du Français Henri-François Imbert, est une élégante tentative (unanimement saluée par la critique française) de rencontre entre deux mondes comme entre le souvenir d'enfance et la réalité d'aujourd'hui. A la recherche d'un ami de son père au Mali, Imbert se laisse heureusement bousculer par ses rencontres qui prennent finalement son film en main. Le voyage se fait initiatique. Le Mauritanien Abderrahmane Sissako part lui aussi à la recherche d'un ami en Angola, dans Rostov-Luanda (1997). Ses rencontres esquissent peu à peu un état des lieux d'un pays en guerre.

Les deux films sont respectueux et respectables. Ils ne s'opposent pas mais se complètent. Imbert filme pour comprendre l'Autre, détour nécessaire pour revenir à lui-même: tout en répondant à ses interrogations de mémoire intérieure, il tente de capter les images d'une réalité, tant la pauvreté des conditions de vie qui ramènent à l'humilité que la qualité de l'accueil et la disponibilité. Sissako filme pour se comprendre lui-même: il connaît trop cette réalité pour vouloir la montrer. Ce sont plutôt les vides qu'il tente de saisir: non pas la guerre mais ses stigmates, ses traces en chacun, en un mot l'absence de paix. Ce n'est pas d'une réalité qu'il cherche à témoigner mais de ce manque, du fait qu'il ne peut la mettre de côté. C'est l'Histoire complexe de l'Afrique qu'il explore sur le mode de l'intime, sa 
propre histoire. Alors que la disponibilité et le sourire des gens qu'il rencontre ramènent Imbert à lui-même, Sissako partage avec eux la même quête angoissée que le temps finit par rendre sereine et déterminée, comme dans La Vie sur terre. C'est cela qu'il filme, car c'est cela sa vérité.

On pourrait en dire autant des Oubliées (1997) de la Togolaise Anne-Laure Folly, qui part elle aussi en Angola en expliquant: "Ce n'est pas pour comprendre cette guerre que je suis partie là-bas, c'est pour rencontrer ses victimes. "Comme dans Femmes du Niger, entre intégrisme et démocratie (1993) et Femmes aux yeux ouverts (1994), elle documente l'expérience intime de l'oppression vécue par les femmes, comme par tous les colonisés, mais aussi leur conscience et leur capacité de rébellion.

Comprenons-nous bien: ce regard n'est intérieur que par la conscience qu'il comporte. "J'ai construit le film avec un regard extérieur", dit Anne-Laure Folly. Elle est partie de Paris en déclarant ne rien connaître rien à l'Angola :

Pour ne pas tromper et ne pas prétendre. Je crois que la plupart des œuvres audiovisuelles sont de l'ordre du regard extérieur. Un Français qui filme l'Auvergne profonde n'y connaît pas forcément grand-chose. Le fait d'être Noire ne m'en donne pas la culture, sortie de mon petit coin d'enfance. Etre Africain ne veut pas dire avoir une culture sur l'Afrique, juste une culture provenant de l'Afrique, contrairement à l'affirmation immodeste et effarante qu'on entend parfois! Être Zoulou ne te donne pas les clefs de la culture yoruba (Barlet, 1997b, p. 13).

Ce regard est une façon de dire "Ouvre tes yeux". C'est d'ailleurs le titre mooré de Pouc Niini (1995) de la Burkinabè Fanta Regina Nacro, dans lequel une femme réagit d'abord avec colère contre l'infidélité de son mari: elle consulte un marabout et fait battre sa rivale. Mais une fois sa révolte passée, elle essaye de comprendre pourquoi son mari lui préfere une courtisane et, chose rarissime, va lui rendre visite pour discuter sans agressivité. Ce pourrait être le titre de son film suivant, Le Truc de Konaté (1998), amusante fable sur la nécessité de mettre des condoms pour se protéger du sida. Ce pourrait aussi 
être le titre de Chef! (1998) de Jean-Marie Teno, édifiante méditation sur les chefs, petits et grands, qui piègent la société camerounaise...

C'est pour sa spécificité qu'il est essentiel que ce regard ne soit pas évincé, dans les programmations des festivals comme à la télévision, par le regard occidental. Celui-ci peut avoir sa valeur, mais il doit rencontrer le regard de l'Autre puisqu'il se trouve qu'il existe, caméra au poing! Seul ce regard du vide, du manque, de l'absence, exprimé par une esthétique correspondante, faite d'incertitude et de fragmentation mais aussi d'engagement, est à même de capter ce monde fissuré de l'Afrique d'aujourd'hui et du vécu du peuple noir. Ce regard intérieur ne se concentre pas sur le combat contre le néocolonialisme. Il étudie de l'intérieur en quoi il affecte les relations humaines, affectives et sociales. Sans doute est-ce à la fois sa force et sa faiblesse, cette weightlessness évoquée par Salman Rushdie: la résistance à la domination ne se fera plus dans de grands combats militants mais dans la responsabilisation de chacun.

Chercheur autonome

\section{NOTES}

1. Propos recueillis lors d'un entretien qui s'est déroulé à Paris en 1995.

2. Propos recueillis au cours d'un entretien à Cannes en 1998 et publiés sur www.africultures.com.

3. Voir à ce sujet l'entretien d'Olivier Barlet avec Youssef El Ftouh, «Ce que filmer veut dire ", Africultures: $n^{\circ} 3$ (1997), p. 9-14. Pour l'analyse de ces préjugés, voir l'article de Nicolas Bancel et Pascal Blanchard, "Sauvage ou assimile? ", Africultures, $\mathrm{n}^{\circ} 25$ (2000), p. 36-43.

4. A ce sujet, voir l'article d'Olivier Barlet, «Postcolonialisme et cinéma: de la différence à la relation, Africultures, $\mathrm{n}^{\circ} 28$ (2000), p. 56-60.

5. A ce sujet, voir les articles d'Olivier Barlet : "La critique occidentale des images d'Afrique ", Africultures, n' 1 (1997), p. 5-11; "Cinémas d'Afrique noire: le nouveau malentendu, Cinémathèque, n' 14 (1998), p. 107-116.

6. La guilde africaine des réalisateurs et producteurs est une association regroupant les cinéastes habitant en France "pour nous parler davantage, échanger nos expériences, afin d'améliorer la qualité de nos films, trouver des solutions pour une meilleure circulation de nos œuvres et enfin mieux défendre nos intérêts " (extrait de l'éditorial de ce premier bulletin). A la première page, on trouve un article intitulé "L'insoutenable "invisibilité" des Noirs en France», cosigné par Balufu Bakupa-Kanyinda, Issa Serge Coelo, Mahamat Saleh Haroun, Mama Keïta, Zéka Laplaine, Fanta Régina Nacro, Jean-Marie Teno, François Woukoache. 
7. Propos recueillis au cours d'un entretien avec José Zéka Laplaine à Paris en 1996 et publiés sur www.africultures.com.

8. Voir à ce sujet l'article d'Olivier Barlet, "Le corps profond des cinémas d'Afrique ", Africultures, n 19 (1999), p. 11-16.

9. Voir également l'entretien de Boniface Mongo-Mboussa avec Jean-Marc Moura dans Africultures, $\mathrm{n}^{\circ} 28$ (2000), p. 14-22.

10. Propos recueillis au cours d'un entretien avec Balufu Bakupa-Kanyinda, à Berlin en février 2000, et publiés sur www.africultures.com.

11. Propos recueillis au cours d'un entretien avec François Woukoache à Cannes en 1999 et publiés sur www.africultures.com.

12. Propos recueillis au Fespaco de Ouagadougou, en février 1999, et publiés sur www.africultures.com.

13. Voir à ce sujet l'article d'Alain Bergala, "Le choix de Godard", Les Cabiers du cinéma, supplément au $\mathrm{n}^{\circ} 537$ (1999).

\section{OUVRAGES CITÉS}

Bah, Ousmane "Achille Mbembe: il nous faut revaloriser la vie". LAutre Afrique, $\mathrm{n}^{\circ} 71$ (1998), p. 50-54.

Bancel, Nicolas et Pascal Blanchard. "Sauvage ou assimilé ?". Africultures, n 25 (2000), p. 36-43.

Barlet, Olivier. "Postcolonialisme et cinéma: de la différence à la relation". Africultures, $\mathrm{n}^{\circ} 28$ (2000), p. 56-60.

Barlet, Olivier "Le corps profond des cinémas d'Afrique ". Africultures, n 19 (1999), p. 11-16.

Barlet, Olivier. "Cinémas d'Afrique noire: le nouveau malentendu". Cinémathèque, $\mathrm{n}^{\circ} 14$ (1998a), p. 107-116.

Barlet, Olivier. "Entretien avec Abderrahmane Sissako". Africultures, n 10 (1998b), p. $99-100$.

Barlet, Olivier. "Entretien avec François Woukoache". Africultures, n” 6 (1998c), p. $40-42$.

Barlet, Olivier. "In memoriam David Achkar». Africultures, n 5 (1998d), p. 8-11.

Barlet, Olivier. "Ce que filmer veut dire». Africultures, $n^{\circ} 3$ (1997a), p. 9-14.

Barlet, Olivier. "Entretien avec Anne-Laure Folly». Africultures, n 2 (1997b), p. $12-$ 14.

Barlet, Olivier. "La critique occidentale des images d'Afrique". Africultures, n 1 $(1997 \mathrm{c})$, p. 5-11.

Barlet, Olivier. Les Cinémas d'Afrique noire: le regard en question. Paris: L'Harmattan, 1996.

Barlet, Olivier. "Nouveaux visages". Ecrans d'Afrique, vol. x, n 11 (1995), p. 17.

Bergala, Alain. "Le choix de Godard». Les Cabiers du cinéma, supplément au n 537 (1999).

Deleuze, Gilles. L'Image-temps. Paris: Minuit, 1985.

Glissant, Édouard. Le Discours antillais. Paris: Gallimard, 1981.

Mongo-Mboussa, Boniface. "Entretien avec Jean-Marc Moura". Africultures, $\mathrm{n}^{\circ} 28$ (2000), p. 14-22.

Moura, Jean-Marc. Littératures francophones et theorie postcoloniale. Paris: PUF, 1999. 